\title{
Cryo-electron tomography of Birbeck granules reveals the molecular mechanism of langerin lattice formation
}

\author{
Authors: Toshiyuki Oda ${ }^{1 *}$, Haruaki Yanagisawa ${ }^{2}$ Hideyuki Shinmori $^{3}$, Youichi Ogawa ${ }^{4}$, \\ and Tatsuyoshi Kawamura ${ }^{4}$
}

Affiliations: ${ }^{1}$ Department of Anatomy and Structural Biology, Graduate School of

Medicine, University of Yamanashi, 1110 Shimokato, Chuo, Yamanashi, 409-3898, Japan. ${ }^{2}$

Department of Cell Biology and Anatomy, Graduate School of Medicine, the University of

Tokyo, 7-3-1 Hongo, Bunkyo-ku, Tokyo, 113-0033, Japan.

${ }^{3}$ Faculty of Life and Environmental Science, University of Yamanashi, 4-4-37 Takeda,

Kofu, Yamanashi, 400-8510, Japan

${ }^{4}$ Department of Dermatology, Faculty of Medicine, University of Yamanashi, 1110

Shimokato, Chuo, Yamanashi, 409-3898, Japan.

*Correspondence to: toda@yamanashi.ac.jp 


\section{Abstract}

Langerhans cells are specialized antigen-presenting cells localized within the epidermis and mucosal tissue. Upon contact with Langerhans cells, pathogens are captured by the C-type lectin langerin and internalized into a structurally unique vesicle known as a Birbeck granule. Although the immunological role of Langerhans cells and Birbeck granules have been extensively studied, the mechanism by which the characteristic zippered membrane structure of Birbeck granules is formed remains elusive. In this study, we observed isolated Birbeck granules using cryo-electron tomography and reconstructed the 3D structure of the repeating unit of the honeycomb lattice of langerin at $6.4 \AA$ resolution. We found that the interaction between the two langerin trimers was mediated by docking the flexible loop at residues 258262 into the secondary carbohydrate-binding cleft. Mutations within the loop inhibited Birbeck granule formation and the internalization of HIV pseudovirus. These findings suggest a molecular mechanism for membrane zippering during Birbeck granule biogenesis and provide insight into the role of langerin in the defense against virual infection. 


\section{Introduction}

The epidermis and mucosa provide the first line of defense against pathogens. In these tissues, specialized antigen-presenting cells called Langerhans cells capture pathogens by recognizing their surface mannose-containing oligosaccharides via the C-type lectin langerin (Valladeau et al., 2003; Doebel et al., 2017). Pathogens internalized via langerin are enclosed in a specialized organelle called a Birbeck granule, which has a central striated lamella between closely apposed membranes (Birbeck et al., 1961; Valladeau et al., 2000;

McDermott et al., 2004; Valladeau et al., 1999). Once pathogens are captured, Langerhans cells undergo maturation and migrate to peripheral lymph nodes, where they present processed antigens to T cells (Merad et al., 2008; de_Jong MA, 2010; Rhodes et al., 2021). Langerin is a type II transmembrane protein that is composed of a cytoplasmic tail, coiledcoil neck region, and carbohydrate recognition domain (CRD) (Stambach and Taylor, 2003; Valladeau et al., 1999; Valladeau et al., 2000) (Figure 1A). Through the coiled-coil neck region, langerin forms a trimer, which is functionally important for oligosaccharide binding (Stambach and Taylor, 2003; Weis and Drickamer, 1996; Weis and Drickamer, 1994). The calcium-dependent carbohydrate-binding capacity of langerin CRD is essential for the 
disintegrate (Stambach and Taylor, 2003; Bartosik, 1992). It has been proposed that the "faceto-face" interaction between the langerin trimers via CRDs confers the characteristic zippered membranes with a central striation of Birbeck granules (Thepaut et al., 2009) (Figure 1B and 1C). However, the precise molecular mechanism of Birbeck granule formation is unknown because of the lack of a high-resolution structure.

Langerin's high affinity for mannose and oligo-mannose allows Langerhans cells to capture a wide range of pathogens, including Saccharomyces cerevisiae, Candida albicans, Mycobacterium leprae, herpes simplex virus, and human immunodeficiency virus-1 (HIV-1) (Tateno et al., 2010; Hunger et al., 2004; de_Jong et al., 2010; de_Witte et al., 2007; Turville et al., 2002). Although Langerhans cells in the mucosa are the initial target of HIV-1 in sexually transmitted infections, the efficiency of Langerhans cell infection is low, requiring high viral loads of HIV-1 to infect cells (Kawamura et al., 2001; Kawamura et al., 2000; Ogawa et al., 2013). The resistance of Langerhans cells to HIV-1 infection is mediated by the efficient internalization of HIV-1 into Birbeck granules and subsequent autophagic degradation (de_Witte et al., 2007; Ribeiro et al., 2016). Therefore, elucidating the mechanism by which HIV-1 is internalized into Birbeck granules is essential to understanding the role of Langerhans cells as a barrier to HIV-1 infection. 
In the present study, we reconstructed the 3D structure of isolated Birbeck granules using cryoelectron tomography and identified a flexible loop region that is crucial for Birbeck granule formation and virus internalization.

\section{Results}

\section{Cryo-electron microscopy of isolated Birbeck granules}

Exogenous expression of langerin in mammalian cell lines readily reproduces the formation

of Birbeck granules (McDermott et al., 2004). We found that the addition of yeast mannan to langerin-expressing cells enhances the formation of large Birbeck granules (Figure 1C). To purify these Birbeck granules, we expressed SNAP-tagged human langerin in 293T cells, biotinylated the tag by incubation with benzylguanine-biotin, and immunoprecipitated the Birbeck granules using streptavidin agarose (Figure S1A and S1B). Under cryo-electron microscopy, the isolated Birbeck granules were observed to be thin lamellar vesicles of assorted sizes, similar to those reported previously (Figure 1D, 1E, and S1C) (Sagebiel and Reed, 1968; Lenormand et al., 2013). Unlike regular 2D crystals of proteins, Birbeck granules appear wavy, suggesting structural heterogeneity. The $2 \mathrm{D}$ classification of the segmented images extracted from the micrographs showed nearly three-fold symmetrical 
arrangements of pores with a diameter of approximately $10 \mathrm{~nm}$; however, no clear crystalline lattice was observed (Figure 1F). Although most Birbeck granules were oriented in approximately the same direction with respect to the beam axis, some granules were twisted, providing different views that were sufficient for 3D reconstruction (Figure S1D).

\section{Subtomogram averaging of the repeating unit of Birbeck granules}

We conducted cryo-electron tomography of the isolated Birbeck granules and reconstructed the repeating units by averaging the subtomograms (Figure 2 and S2A). The initial density map (Figure 2A) shows a langerin trimer (blue) with three inverted trimers (red) attached to the CRDs. However, because of the flexibility between each langerin trimer, the resolution was limited to $\sim 24 \AA$. To improve the resolution, we generated a mask enclosing the central langerin trimer and three attached CRDs and performed focused refinement and 3D classification (Figure 2B). The resolution was improved to $6.4 \AA$ (Figure S2B), which clearly visualized the alpha helices and enabled us to generate a model based on the previously reported crystal structure of the langerin trimer (Feinberg et al., 2010). We then performed a two-body refinement to visualize the spatial relationship between the two opposing langerin trimers (Nakane et al., 2018). Based on this refinement (Figure 2C), we built a honeycomb 
lattice model (Figure 2D and Movie S1), which agrees with the striation of the Birbeck granules observed by ultra-thin section electron microscopy and the $\sim 10 \mathrm{~nm}$ pores visualized in the 2D classification images (Figure 1C and 1F) (Birbeck et al., 1961). However, in reality, the honeycomb lattice is non-uniform because of the structural heterogeneity.

\section{Interaction between the two langerin trimers via flexible loops}

An in-depth analysis of the interaction region between the two CRDs (Figure 3) revealed that the interaction is mediated by a loop at residues $258-262$, which is highly flexible in the crystal structure (Feinberg et al., 2010). The interaction between the two CRDs appears to be maintained by docking the loops of two langerin molecules into each other's lateral cleft, which constitutes the secondary carbohydrate-binding site (Figure 3A, pink circles) (Chatwell et al., 2008). The interaction between the two CRDs is flexible because the first eigenvector of the multi-body analysis represents an approximately $30^{\circ}$ tilting motion with the loop as the fulcrum (Figure 3B, and Movie S2). Interestingly, loop 258-262 of the inverted molecule appears to participate in the secondary carbohydrate-binding site of the upright molecule (Figure 3A). A previous study found that the secondary calcium-independent binding site has a lower affinity for carbohydrate ligands than the primary calcium-dependent 
binding site (Chatwell et al., 2008). The docked loop 258-262 may provide additional interactions with the hydroxyl group of mannose via Glu261 and Asp263, thus increasing the affinity of the secondary binding site when two langerin trimers form a complex. Furthermore, the Trp264Arg mutation is associated with a lack of Birbeck granules (Verdijk et al., 2005;

Figdor et al., 2002). Trp264 may also be involved in docking the loop into the cleft; however, it is more likely that disruption of the hydrophobic core of the CRD is responsible for this defect in Birbeck granule formation (Chatwell et al., 2008).

\section{Loop 258-262 is essential for Birbeck granule formation}

To investigate the importance of loop 258-262 in the biogenesis of Birbeck granules, we mutated three residues in the loop: Met260Ala, Glu261Arg, and Asp263Lys, and generated three mutated langerin constructs, each carrying one (MRGD), two (MRGK), or three mutations (ARGK). We expressed these three langerin mutants in 293T cells and induced

Birbeck granule formation by adding yeast mannan (Figure 4A). Under ultra-thin section microscopy, mannan-induced Birbeck granules usually formed a cluster, and wild-type langerin produced Birbeck granules a few micrometers in length (Figure 4A, MEGD WT). As the number of mutations increased, the length of Birbeck granules decreased. With two 
point mutations (MRGK), some cross-sections became spherical rather than rod-shaped, suggesting that the Birbeck granules failed to extend. With three mutations (ARGK), clusters of Birbeck granules were not observed, with isolated individual granules observed instead.

The length of individual Birbeck granules and the total length of granules per area were measured to confirm the above observations (Figure 4B and 4C). These results demonstrate the importance of loop 258-262 in the biogenesis of Birbeck granules.

\section{Loop 258-262 is crucial for the internalization of HIV pseudovirus}

To investigate the impact of the mutations in loop 258-262 on virus internalization by Birbeck granules, we added lentiviruses pseudo-typed with the HIV-1 env protein to langerinexpressing $293 \mathrm{~T}$ cells. After $30 \mathrm{~min}$ of incubation, we retrieved viruses attached to the surface langerin by washing with EDTA buffer, removed non-specifically attached viruses by trypsinization, and isolated the Birbeck granules using streptavidin-agarose (Figure 5). A langerin mutant that lost its calcium-binding capacity (Stambach and Taylor, 2003) was used as the negative control (Figure 5, lectin (-)). The HIV pseudoviruses attached to both wildtype and ARGK mutant langerin and the attachment was abolished by the addition of mannan

(Figure 5A). This indicated that the mutation of the loop did not disrupt the binding of the 
glycosylated envelope proteins to the primary carbohydrate-binding sites. In contrast, the internalization of the pseudovirus was significantly inhibited by the ARGK mutation (Figure 5B and $5 \mathrm{C}$ ). These results suggest that disruption of Birbeck granule formation by the mutation of loop 258-262 inhibited the internalization of HIV pseudovirus.

\section{Discussion}

\section{Virus internalization mechanism of Birbeck granules}

In the present study, we reconstructed the 3D structure of isolated Birbeck granules and found that docking of the flexible loop 258-262 into the secondary carbohydrate-binding cleft is essential for assembly of the langerin lattice. In agreement with this observation, we showed that mutations introduced into the loop inhibited Birbeck granule formation and internalization of HIV pseudovirus. Based on the structure of the glycosylated HIV env protein (Stewart-Jones et al., 2016; Pan et al., 2020), we propose a model of HIV internalization by langerin (Figure 6A and 6B). A previous study proposed that N-linked high-mannose oligosaccharides bind to langerin at both primary and secondary carbohydratebinding sites (Chatwell et al., 2008). Our model requires bending of the coiled-coil necks of the two langerin trimers to form a complex with HIV-1 env via the high-mannose 
oligosaccharide (Figure 6B). Although the triple coiled-coil structure of the neck domain does not seem to bend strongly, the coiled-coil prediction profile suggests that the N-terminal part of the neck does not necessarily form a rigid triple coiled coil (Lupas et al., 1991) (Figure

S1E). Thus, we speculate that the neck region of the langerin trimer is flexible and swings on the cell surface, allowing loop 258-262 of one trimer to dock into the cleft of an adjacent trimer. Once the triad structure, consisting of two langerin trimers and one env protein, is formed, the elasticity of the neck region can deform the cell membrane and initiate invagination. The formation of clathrin pits may also be involved in membrane invagination, because the cytoplasmic ends of Birbeck granules are coated with clathrin (Mc et al., 2002). Additionally, it is possible that other intracellular mechanisms such as the actin cytoskeleton, are involved in this membrane deformation process because annexin A2 is required for the proper formation of Birbeck granules (Thornton et al., 2020).

\section{Significance of langerin-specific secondary carbohydrate-binding cleft}

It is noteworthy that the docking of loop 258-262 into the secondary calcium-independent carbohydrate-binding cleft, which is not found in other C-type lectins (Chatwell et al., 2008), mediates the assembly of the langerin lattice. In DC-SIGN, which is another C-type lectin 
expressed in dendritic cells, and is closely related to langerin, the region corresponding to the secondary carbohydrate-binding site of langerin forms a shallow pocket, that is occupied by two calcium ions (Figure 7A) (Guo et al., 2004; Geijtenbeek et al., 2000; Geijtenbeek et al., 2000). This structural difference between langerin and DC-SIGN may account for the inability of DC-SIGN to form Birbeck granules despite its high sequence similarity to langerin (Cambi et al., 2009). Another characteristic of the secondary carbohydrate-binding site of langerin is its weak affinity for mannose and maltose (Chatwell et al., 2008). This may reflect that the sugar-binding surface of langerin CRD is less negatively charged than that of DC-SIGN CRD (Figure 7A) (Chatwell et al., 2008). Moreover, the interaction between the secondary binding site and mannose/maltose is mainly mediated by backbone amide and carbonyl groups (Chatwell et al., 2008). It is possible that the side chains of Glu261 and Asp263 of loop 258-262 docked into the cleft of an adjacent langerin CRD provide the negative charge required for stabilizing oligo-mannose bound to the secondary binding site (Figure 7A and B). In agreement with this hypothesis, in the present study the Asp263Lys mutation caused a significant defect in Birbeck granule formation. The secondary carbohydrate-binding site may only be fully functional when two langerin trimers are bound to each other (Figure 6 and 7B). The effect of the Met260Ala mutation on Birbeck granule 
formation was unexpected, because the mutation was supposed to be silent in terms of side chain charge. The observed structural variation of the langerin trimer complex suggests that the side chain of Met260 acts as a hook to stabilize the interface between the two intersecting loops.

Our model of Birbeck granule formation suggests that oligosaccharide ligands are captured by the lateral surface of langerin (Figure 6). In contrast, DC-SIGN binds to dengue virus on its upper surface (Pokidysheva et al., 2006). Considering that DC-SIGN serves as an attachment receptor for various viruses but not as an entry receptor (Bermejo-Jambrina et al., 2018; Lempp et al., 2021), the possible structural coupling of lateral ligand binding and internalization (Figure 6B) implies that langerin functions as a more efficient entry receptor for viruses than DC-SIGN or other C-type lectins. Further studies are needed to investigate the physiological significance of the unique carbohydrate-binding mechanism of langerin and the honeycomb lattice structure of Birbeck granules. 


\section{Methods}

\section{Isolation of Birbeck granules}

The cDNA of human langerin was obtained from the Mammalian Gene Collection library

(Dnaform Inc., Yokohama, Japan) and was subcloned into pcDNA3.1-IRES-GFP (Schaefer

et al., 2008). A codon-optimized sequence coding SNAP-3 $\times$ HA-HRV3C cleavage site

(obtained from ThermoFisher Scientific, Waltham, MA) was inserted immediately before the

start codon of the langerin sequence (Cole, 2013). $\mathrm{O}^{6}$-benzylguanine-biotin was obtained by

reacting $16 \mathrm{mM} \mathrm{O}^{6}$-benzylguanine-amine (Accela ChemBio, Shanghai, China) with $50 \mathrm{mM}$

biotin-AC5-Osu (Dojindo, Kumamoto, Japan) and $30 \mathrm{mM}$ triethylamine in

dimethylformamide for 24 hours at $30^{\circ} \mathrm{C}$. Lenti-X 293T cells (TakaraBio, Tokyo, Japan) were

transfected with the langerin-expression plasmid by electroporation using ECM630

electroporator (BTX, Holliston, MA) and seeded onto the polyethylenimine-coated dish (PEI,

obtained from Fujifilm Wako Chemicals, Osaka, Japan). After 48-hour culturing in

$\mathrm{DMEM}+10 \%$ fetal bovine serum $(\mathrm{FBS})$, the culture medium was replaced with serum-free

DMEM containing $10 \mu \mathrm{M} \mathrm{O}^{6}$-benzylguanine-biotin and $10 \mu \mathrm{g} / \mathrm{ml}$ yeast mannan (Nacalai

tesque, Kyoto, Japan) and the cells were incubated for $30 \mathrm{~min}$ at $37^{\circ} \mathrm{C}$. The cells were then

collected by scraping and washed five times with isotonic HMC buffer (Hepes $30 \mathrm{mM}, \mathrm{pH}$ 
7.2, $150 \mathrm{mM} \mathrm{NaCl}, 2 \mathrm{mM} \mathrm{MgCl}, 2 \mathrm{mM} \mathrm{CaCl}_{2}$, protease inhibitor cocktail (Nacalai tesque)).

The washed cells were homogenized with a Dounce glass homogenizer and were further disrupted by sonication for 8 seconds using a Q125 sonicator (Qsonica, Newtown, CT). The cell homogenate was loaded on top of a discontinuous Opti-prep density gradient (10-20$40 \%$ layers, ThermoFisher Scientific) and ultracentrifuged for 1 hour at $100,000 \times \mathrm{g}$. The fraction at the interface of $20 / 40 \%$ layers was collected and diluted 10 -fold with HMC buffer and was again disrupted by sonication for $2 \min$ (120 cycles of 1 -sec pulse and 3 -sec wait) at $4^{\circ} \mathrm{C}$. Large debris was removed by filtration through a $20 \mu \mathrm{m}$-pore polyethylene mesh (GL Science, Tokyo, Japan) and the cell lysate was incubated with a $30 \mu 1$ slurry of streptavidin agarose (Solulink, San Diego, CA) overnight at $4^{\circ} \mathrm{C}$. The streptavidin agarose beads were washed five times with HMC buffer and the bound Birbeck granules were eluted by overnight digestion using home-made E. coli overexpressed His $\times 6-\mathrm{HRV} 3 \mathrm{C}$ protease in HMC buffer at $4^{\circ} \mathrm{C}$. After digestion and elution, HRV3C protease was removed by incubating the elute with Ni-NTA resin (Qiagen, Düsseldorf, Germany). About $50 \mu 1$ of elute containing released Birbeck granules (approximately $0.05 \mathrm{mg} / \mathrm{ml}$ ) were obtained from twelve 100-mm dishes of cell culture. 


\section{Cryo-electron microscopy of isolated Birbeck granules}

$5 \mu 1$ of the purified Birbeck granules was applied onto freshly glow-discharged holey carbon

grids, Quantifoil R1.2/1.3 Cu/Rh 200 mesh (Quantifoil Micro Tools GmbH, Großlöbichau,

Germany), blotted from the backside to concentrate the granules by filtration through the

holes, and $4 \mu$ l of HMC buffer containing cytochrome $c$-stabilized 15-nm colloidal gold (BBI

Solutions) was applied for attachment of fiducial markers (Oda et al., 2015). Grids were

blotted from the backside for 10 seconds at $4^{\circ} \mathrm{C}$ under $99 \%$ humidity and plunge frozen in

liquid ethane using Vitrobot Mark IV (Thermo Fisher Scientific). We removed one of the

blotting arms for one-side blotting because Birbeck granules would be absorbed onto the

filter paper. Images were recorded using a Titan Krios G3i microscope at the University of

Tokyo (Thermo Fisher Scientific) at $300 \mathrm{keV}$, a Gatan Quantum-LS Energy Filter (Gatan,

Pleasanton, CA) with a slit width of $20 \mathrm{eV}$, and a Gatan K3 Summit direct electron detector

in the electron counting mode. The nominal magnification was set to $33,000 \times$ with a physical

pixel size of $2.67 \AA /$ pixel. Movies were acquired using the SerialEM software (Mastronarde,

2005), and the target defocus was set to 3-6 $\mu \mathrm{m}$. The angular range of the tilt series was from

$-60^{\circ}$ to $60^{\circ}$ with $3.0^{\circ}$ increments. Each movie was recorded for $0.74 \mathrm{sec}$ with a total dose of

1.24 electrons $/ \AA^{2}$ and subdivided into 20 frames. The total dose for one tilt series acquisition 
is thus 50 electrons $/ \AA^{2}$.

\section{Data processing}

Movies were subjected to beam-induced motion correction using MotionCor2 (Zheng et al., 2017), and tilt series images were aligned, CTF corrected, and back-projected to reconstruct

3D tomograms using the IMOD software package (Kremer et al., 1996). For the subsequent

analysis using Relion-4.0 (Kimanius et al., 2021), the tomogram positioning step was skipped

to keep the angle offset and the Z-shift zero. Tomograms were $4 \times$ binned to reduce loads of

the calculation. The areas of the Birbeck granules were manually selected using the $3 \mathrm{dmod}$

model tool (Kremer et al., 1996), and the center coordinates for the subtomograms were

determined by placing points at 8-pixel $(\sim 8.5 \mathrm{~nm})$ intervals within the selected area using a

custom-made Ruby script (Metlagel et al., 2007). For twisting Birbeck granules (Figure S1D),

center points were picked manually by selecting the mid-portion of the lamella. Volumes with

$32 \times 32 \times 32$ pixel-dimensions were extracted from $8 \times$ binned tomograms and were averaged

using the PEET software suite (Nicastro et al., 2006). A randomly selected subtomogram was

used for the initial reference. Alignments were repeated five times for $8 \times$ binned and twice

for $4 \times$ binned tomograms with $64 \times 64 \times 64$ pixel-dimensions. The coordinates and the rotation 
angles of the aligned subtomograms were exported to Relion-4.0. Initial pseudosubtomograms were generated with the box size of 128 pixels, the cropped box size of 64 pixels, and the binning factor of 4 . The subtomograms were $3 \mathrm{D}$ refined using a spherical mask of $320-\AA$ diameter. After $3 \mathrm{D}$ classification, $2 \times$ binned pseudosubtomograms were generated with the box size of 256 pixels and the cropped box size of 128 pixels based on the refined coordinates and angles and were 3D refined using the same spherical mask. Finally, un-binned pseudosubtomograms were generated with a box size of 256 pixels and a cropped box size of 128 pixels. 3D refinement was done using a tighter mask enclosing the central upright langerin molecule and three bound CRDs, and low-resolution subtomograms were removed by $3 \mathrm{D}$ classification. The alignment was further refined using CTF refinement and frame alignment. We initially refined the surrounding inverted langerin molecule by 3D refinement masking out the central upright molecule, but the refinement was biased toward the center. To improve the map quality of the inverted molecule, we applied 3D multi-body refinement (Nakane et al., 2018), which successfully compensated for the flexibility between the upright and the inverted molecules. For model building, the crystal structure of langerin trimer (Feinberg et al., 2010) (PDB ID: 3KQG) was fitted into the map using UCSF Chimera (Pettersen et al., 2004). Then, the fitted model was further refined using PHENIX real-space 
refinement tool, and the refined model was validated using the comprehensive validation tool

(Adams et al., 2010). The Coulombic surfaces rendering was conducted using UCSF

Chimera and other model presentations, surface rendering, and morph movie generation were

conducted using ChimeraX (Goddard et al., 2018). The data processing and the statistics of the model validation are summarized in Figure S2A and Table S1.

\section{Ultrathin section electron microscopy of Birbeck granules}

Pellets of $293 \mathrm{~T}$ cells with mannan-induced Birbeck granules were fixed with 4\% paraformaldehyde for 1 hour at $4{ }^{\circ} \mathrm{C}$ and stained with $1 \%$ osmium tetroxide and subsequently with $1 \%$ uranium acetate. After dehydration in ethanol and acetone, the samples were embedded in Quetol 812 resin (Nissin EM, Tokyo, Japan). 60-nm ultrathin sections were cut using a ULTRACUT microtome (Reichert Leica) and mounted onto Formvar-coated copper grid. Images were recorded using a JEM-2100F microscope (JEOL, Tokyo, Japan) at University of Yamanashi operated at $200 \mathrm{keV}$ equipped with an F216 CMOS camera (TVIPS $\mathrm{GmbH}$, Gauting, Germany). Length of Birbeck granules were measured using Fiji software (Schindelin et al., 2012), and plots were generated using PlotsOfData web tool (Postma and Goedhart, 2019). For total length of Birbeck granules per $100 \mu \mathrm{m}^{2}$, cross sections of cells 
with nuclei of $>5 \mu \mathrm{m}$ in diameter were selected, and the sum of the lengths of Birbeck

granules within the cells were measured. The areas of the cross sections were also measured using Fiji software. $100 \mu \mathrm{m}^{2}$ corresponds to the approximate mean of the cross-sectional area of one cell.

\section{Lentivirus preparation}

Lentiviruses pseudotyped with HIV-1 env were prepared by transfecting the Lenti-X 293T cells with pCMV-dR8.3 $\Delta$ vpr (Addgene plasmid \#8455), pLOX-CW-tdTomato, and pCEP4AD8gp160 (Adgene plasmid \#123260) (Stewart et al., 2003; Heredia et al., 2019). The vector pLOX-CW-tdTomato was generated by replacing the GFP sequence of pLOX-CWgfp (Addgene plasmid \#12241) (Salmon et al., 2000) with the tdTomato sequence derived from pCDH-EF1-Luc2-P2A-tdTomato, a gift from Kazuhiro Oka (Addgene plasmid \# 72486). The transfections were carried out using the PEI-method with the ratio at PEI: dR8.3: tdTomato: gp160 $=4: 1: 1: 1.16$ hours post-transfection, the cells were washed three times with PBS and incubated in a fresh medium for another 32 hours. The virus-containing medium was collected and removed cell debris by a $1,000 \times \mathrm{g}$ centrifuge and a $0.45 \mu \mathrm{m}$ filtration (Millipore).

The obtained supernatants were immediately used for subsequent assays. 


\section{Virus attachment and internalization assay}

293T cells were transfected with wild type langerin, the ARGK mutant, and, as a negative control, a lectin (-) mutant lacking calcium-binding ability. The lectin (-) langerin mutant was generated by introducing three point-mutations at the calcium-binding site: Glu285Lys; Asn287Lys; and Glu293Lys (Chatwell et al., 2008). 48 hours post-transfection, the cells were pre-treated with or without $10 \mu \mathrm{g} / \mathrm{ml}$ yeast mannan for $5 \mathrm{~min}$ at $37^{\circ} \mathrm{C}$. The cells were then incubated in the pseudovirus-containing supernatants plus $10 \mu \mathrm{M} \mathrm{O}^{6}$-benzylguanine-biotin and $10 \mu \mathrm{g} / \mathrm{ml}$ DEAE-dextran (Sigma Aldrich, St. Louis, MO) with or without $10 \mu \mathrm{g} / \mathrm{ml}$ yeast mannan for $30 \mathrm{~min}$ at $37^{\circ} \mathrm{C}$. After the incubation, supernatant containing unbound viruses were collected. The cells were then washed with TBS-Ca buffer $(20 \mathrm{mM}$ Tris-HCl pH 7.4, $150 \mathrm{mM} \mathrm{NaCl}, 5 \mathrm{mM} \mathrm{CaCl}_{2}$ ) three times, and the viruses attached to langerin at the cell surface was retrieved by washing with TBS-EDTA buffer $(20 \mathrm{mM}$ Tris-HCl pH 7.4, $150 \mathrm{mM}$ $\mathrm{NaCl}, 10 \mathrm{mM}$ EDTA). The collected supernatant of the medium and the TBS-EDTA buffer were centrifuged at $1,000 \times \mathrm{g}$ for $5 \mathrm{~min}$ to remove cell debris, and the viruses were sedimented by ultra-centrifugation at $100,000 \times \mathrm{g}$ for 3 hours. After the TBS-EDTA washing, nonspecifically bound viruses were removed by trypsinization using $0.25 \%(\mathrm{w} / \mathrm{v})$ trypsin and 
$1 \mathrm{mM}$ EDTA for $5 \mathrm{~min}$ at $37^{\circ} \mathrm{C}$ as previously described (Burkard et al., 2014). Detached cells were washed twice with DMEM $+10 \% \mathrm{FBS}$, and five times with TBS-Ca. Internalized viruses within Birbeck granules were isolated from the washed cells by streptavidin-agarose precipitation as described above. Viruses, langerin, and tubulin were detected by immunoblotting using HIV-1 gag p24 antibody (1:1000 dilution, MAB7360, mouse monoclonal, R\&D systems, Minneapolis, MN), human CD207 antibody (1:1000 dilution, 11841-1-AP, rabbit polyclonal, Proteintech Group Inc., Rosemont, IL), and tubulin antibody (1:3000 dilution, B-512, mouse monoclonal, Sigma-Aldrich), respectively. The p24 antibody detected unprocessed (Pr55 ${ }^{\mathrm{gag}}$ ) and fully processed (p24) capsid proteins (Gonelli et al., 2019). Band intensities were measured using ImageJ (Schneider et al., 2012). 


\section{Acknowledgements}

This research is partially supported by Platform Project for Supporting Drug Discovery and

Life Science Research (Basis for Supporting Innovative Drug Discovery and Life Science

Research (BINDS)) from Japan Agency for Medical Research and Development (AMED)

under Grant Number JP19am0101115. This research was conducted using the Fujitsu

PRIMERGY CX400M1/CX2550M5 (Oakbridge-CX) and the SGI Rackable C2112-

4GP3/C1102-GP8 (Reedbush-H/L) in the Information Technology Center, The University of

Tokyo. This work was supported by the Takeda Science Foundation (to T.O.), the Daiichi

Sankyo Foundation of Life Science (to T.O.), the Japan Society for the Promotion of Science

(KAKENHI Grant number JP21H02654 to T.O.), and the Naito Foundation (to T.O.).

Molecular graphics and analyses performed with UCSF ChimeraX, developed by the

Resource for Biocomputing, Visualization, and Informatics at the University of California,

San Francisco, with support from National Institutes of Health R01-GM129325 and the

Office of Cyber Infrastructure and Computational Biology, National Institute of Allergy and

Infectious Diseases. 


\section{Author contributions}

T.O. conceived the project and designed the experiments. T.O., H.Y., and H.S. performed the experiments. T.O., and H.Y. analyzed the data. T.O., H.Y., Y.O., and T.K. wrote the manuscript.

\section{Data availability}

The map and the model will be available on the EMDB upon publication under the following accession numbers: EMD-32906, and PDB 7WZ8. 


\section{References}

Birbeck, M.S., Breathnach, A.S., and Everall, J.D. 1961. An electron microscope study of basal melanocytes and high-level clear cells (Langerhans cells) in vitiligo. J. Invest.

Dermatol. 37, 51-63.

Sagebiel, R.W., and Reed, T.H. 1968. Serial reconstruction of the characteristic granule of the Langerhans cell. J. Cell. Biol. 36, 595-602.

Lupas, A., Van, Dyke M., and Stock, J. 1991. Predicting coiled coils from protein sequences. Science. 252, 1162-1164.

Bartosik, J. 1992. Cytomembrane-derived Birbeck granules transport horseradish peroxidase to the endosomal compartment in the human Langerhans cells. J. Invest.

Dermatol. 99, 53-58.

Weis, W.I., and Drickamer, K. 1994. Trimeric structure of a C-type mannose-binding protein. Structure. 2, 1227-1240.

Kremer, J.R., Mastronarde, D.N., and McIntosh, J.R. 1996. Computer visualization of three-dimensional image data using IMOD. J. Struct. Biol. 116, 71-76.

Weis, W.I., and Drickamer, K. 1996. Structural basis of lectin-carbohydrate recognition. 
Annu. Rev. Biochem. 65, 441-473.

Valladeau, J., Duvert-Frances, V., Pin, J.J., Dezutter-Dambuyant, C., Vincent, C.,

Massacrier, C., Vincent, J., Yoneda, K., Banchereau, J., Caux, C. et al. 1999. The

monoclonal antibody DCGM4 recognizes Langerin, a protein specific of Langerhans

cells, and is rapidly internalized from the cell surface. Eur. J. Immunol. 29, 2695-2704.

Geijtenbeek, T.B., Krooshoop, D.J., Bleijs, D.A., van, Vliet S.J., van, Duijnhoven G.C.,

Grabovsky, V., Alon, R., Figdor, C.G., and van, Kooyk Y. 2000. DC-SIGN-ICAM-2

interaction mediates dendritic cell trafficking. Nat. Immunol. 1, 353-357.

Geijtenbeek, T.B., Kwon, D.S., Torensma, R., van, Vliet S.J., van, Duijnhoven G.C.,

Middel, J., Cornelissen, I.L., Nottet, H.S., KewalRamani, V.N., Littman, D.R. et al. 2000.

DC-SIGN, a dendritic cell-specific HIV-1-binding protein that enhances trans-infection of

T cells. Cell. 100, 587-597.

Kawamura, T., Cohen, S.S., Borris, D.L., Aquilino, E.A., Glushakova, S., Margolis, L.B.,

Orenstein, J.M., Offord, R.E., Neurath, A.R., and Blauvelt, A. 2000. Candidate

microbicides block HIV-1 infection of human immature Langerhans cells within

epithelial tissue explants. J. Exp. Med. 192, 1491-1500.

Salmon, P., Oberholzer, J., Occhiodoro, T., Morel, P., Lou, J., and Trono, D. 2000. 
Reversible immortalization of human primary cells by lentivector-mediated transfer of specific genes. Mol. Ther. 2, 404-414.

Valladeau, J., Ravel, O., Dezutter-Dambuyant, C., Moore, K., Kleijmeer, M., Liu, Y.,

Duvert-Frances, V., Vincent, C., Schmitt, D., Davoust, J. et al. 2000. Langerin, a novel Ctype lectin specific to Langerhans cells, is an endocytic receptor that induces the formation of Birbeck granules. Immunity. 12, 71-81.

Kawamura, T., Qualbani, M., Thomas, E.K., Orenstein, J.M., and Blauvelt, A. 2001. Low levels of productive HIV infection in Langerhans cell-like dendritic cells differentiated in the presence of TGF-beta1 and increased viral replication with CD40 ligand-induced maturation. Eur. J. Immunol. 31, 360-368.

Figdor, C.G., van, Kooyk Y., and Adema, G.J. 2002. C-type lectin receptors on dendritic cells and Langerhans cells. Nat. Rev. Immunol. 2, 77-84.

Mc, Dermott R., Ziylan, U., Spehner, D., Bausinger, H., Lipsker, D., Mommaas, M., Cazenave, J.P., Raposo, G., Goud, B., de, la Salle H. et al. 2002. Birbeck granules are subdomains of endosomal recycling compartment in human epidermal Langerhans cells, which form where Langerin accumulates. Mol. Biol. Cell. 13, 317-335.

Turville, S.G., Cameron, P.U., Handley, A., Lin, G., Pohlmann, S., Doms, R.W., and 
Cunningham, A.L. 2002. Diversity of receptors binding HIV on dendritic cell subsets. Nat.

Immunol. 3, 975-983.

Stambach, N.S., and Taylor, M.E. 2003. Characterization of carbohydrate recognition by

langerin, a C-type lectin of Langerhans cells. Glycobiology. 13, 401-410.

Stewart, S.A., Dykxhoorn, D.M., Palliser, D., Mizuno, H., Yu, E.Y., An, D.S., Sabatini,

D.M., Chen, I.S., Hahn, W.C., Sharp, P.A. et al. 2003. Lentivirus-delivered stable gene

silencing by RNAi in primary cells. RNA. 9, 493-501.

Valladeau, J., Dezutter-Dambuyant, C., and Saeland, S. 2003. Langerin/CD207 sheds light

on formation of birbeck granules and their possible function in Langerhans cells.

Immunol. Res. 28, 93-107.

Guo, Y., Feinberg, H., Conroy, E., Mitchell, D.A., Alvarez, R., Blixt, O., Taylor, M.E.,

Weis, W.I., and Drickamer, K. 2004. Structural basis for distinct ligand-binding and

targeting properties of the receptors DC-SIGN and DC-SIGNR. Nat. Struct. Mol. Biol.

11, 591-598.

Hunger, R.E., Sieling, P.A., Ochoa, M.T., Sugaya, M., Burdick, A.E., Rea, T.H., Brennan,

P.J., Belisle, J.T., Blauvelt, A., Porcelli, S.A. et al. 2004. Langerhans cells utilize CD1a and

langerin to efficiently present nonpeptide antigens to T cells. J. Clin. Invest. 113, 701- 
708.

McDermott, R., Bausinger, H., Fricker, D., Spehner, D., Proamer, F., Lipsker, D.,

Cazenave, J.P., Goud, B., De, La Salle H., Salamero, J. et al. 2004. Reproduction of

Langerin/CD207 traffic and Birbeck granule formation in a human cell line model. $J$.

Invest. Dermatol. 123, 72-77.

Pettersen, E.F., Goddard, T.D., Huang, C.C., Couch, G.S., Greenblatt, D.M., Meng, E.C., and Ferrin, T.E. 2004. UCSF Chimera--a visualization system for exploratory research and analysis. J. Comput. Chem. 25, 1605-1612.

Mastronarde, D.N. 2005. Automated electron microscope tomography using robust prediction of specimen movements. J. Struct. Biol. 152, 36-51.

Verdijk, P., Dijkman, R., Plasmeijer, E.I., Mulder, A.A., Zoutman, W.H., Mieke,

Mommaas A., and Tensen, C.P. 2005. A lack of Birbeck granules in Langerhans cells is associated with a naturally occurring point mutation in the human Langerin gene. $J$.

Invest. Dermatol. 124, 714-717.

Nicastro, D., Schwartz, C., Pierson, J., Gaudette, R., Porter, M.E., and McIntosh, J.R. 2006.

The molecular architecture of axonemes revealed by cryoelectron tomography. Science.

313, 944-948. 
Pokidysheva, E., Zhang, Y., Battisti, A.J., Bator-Kelly, C.M., Chipman, P.R., Xiao, C.,

Gregorio, G.G., Hendrickson, W.A., Kuhn, R.J., and Rossmann, M.G. 2006. Cryo-EM

reconstruction of dengue virus in complex with the carbohydrate recognition domain of

DC-SIGN. Cell. 124, 485-493.

de_Witte, L., Nabatov, A., Pion, M., Fluitsma, D., de, Jong M.A., de, Gruij1 T., Piguet, V., van, Kooyk Y., and Geijtenbeek, T.B. 2007. Langerin is a natural barrier to HIV-1

transmission by Langerhans cells. Nat. Med. 13, 367-371.

Metlagel, Z., Kikkawa, Y.S., and Kikkawa, M. 2007. Ruby-Helix: an implementation of

helical image processing based on object-oriented scripting language. J. Struct. Biol. 157,

95-105.

Chatwell, L., Holla, A., Kaufer, B.B., and Skerra, A. 2008. The carbohydrate recognition domain of Langerin reveals high structural similarity with the one of DC-SIGN but an additional, calcium-independent sugar-binding site. Mol. Immunol. 45, 1981-1994.

Merad, M., Ginhoux, F., and Collin, M. 2008. Origin, homeostasis and function of Langerhans cells and other langerin-expressing dendritic cells. Nat. Rev. Immunol. 8, 935947.

Schaefer, M.R., Williams, M., Kulpa, D.A., Blakely, P.K., Yaffee, A.Q., and Collins, K.L. 
2008. A novel trafficking signal within the HLA-C cytoplasmic tail allows regulated expression upon differentiation of macrophages. J. Immunol. 180, 7804-7817.

Cambi, A., Beeren, I., Joosten, B., Fransen, J.A., and Figdor, C.G. 2009. The C-type lectin

DC-SIGN internalizes soluble antigens and HIV-1 virions via a clathrin-dependent

mechanism. Eur. J. Immunol. 39, 1923-1928.

Thepaut, M., Valladeau, J., Nurisso, A., Kahn, R., Arnou, B., Vives, C., Saeland, S., Ebel,

C., Monnier, C., Dezutter-Dambuyant, C. et al. 2009. Structural studies of langerin and

Birbeck granule: a macromolecular organization model. Biochemistry. 48, 2684-2698.

Adams, P.D., Afonine, P.V., Bunkoczi, G., Chen, V.B., Davis, I.W., Echols, N., Headd,

J.J., Hung, L.W., Kapral, G.J., Grosse-Kunstleve, R.W. et al. 2010. PHENIX: a

comprehensive Python-based system for macromolecular structure solution. Acta.

Crystallogr. D. Biol. Crystallogr. 66, 213-221.

de_Jong, M.A., de, Witte L., Taylor, M.E., and Geijtenbeek, T.B. 2010. Herpes simplex

virus type 2 enhances HIV-1 susceptibility by affecting Langerhans cell function. $J$.

Immunol. 185, 1633-1641.

de_Jong MA, Geijtenbeek T.B. 2010. Langerhans cells in innate defense against pathogens.

Trends. Immunol. 31, 452-459. 
Feinberg, H., Powlesland, A.S., Taylor, M.E., and Weis, W.I. 2010. Trimeric structure of langerin. J. Biol. Chem. 285, 13285-13293.

Tateno, H., Ohnishi, K., Yabe, R., Hayatsu, N., Sato, T., Takeya, M., Narimatsu, H., and Hirabayashi, J. 2010. Dual specificity of Langerin to sulfated and mannosylated glycans via a single C-type carbohydrate recognition domain. J. Biol. Chem. 285, 6390-6400.

Schindelin, J., Arganda-Carreras, I., Frise, E., Kaynig, V., Longair, M., Pietzsch, T., Preibisch, S., Rueden, C., Saalfeld, S., Schmid, B. et al. 2012. Fiji: an open-source platform for biological-image analysis. Nat. Methods. 9, 676-682.

Schneider, C.A., Rasband, W.S., and Eliceiri, K.W. 2012. NIH Image to ImageJ: 25 years of image analysis. Nat. Methods. 9, 671-675.

Cole, N.B. 2013. Site-specific protein labeling with SNAP-tags. Curr. Protoc. Protein. Sci.

73, 30.1.1-30.1.16.

Lenormand, C., Spiegelhalter, C., Cinquin, B., Bardin, S., Bausinger, H., Angenieux, C.,

Eckly, A., Proamer, F., Wall, D., Lich, B. et al. 2013. Birbeck granule-like "organized smooth endoplasmic reticulum" resulting from the expression of a cytoplasmic YFP-tagged langerin. PLoS. One. 8, e60813.

Ogawa, Y., Kawamura, T., Matsuzawa, T., Aoki, R., Gee, P., Yamashita, A., Moriishi, K., 
Yamasaki, K., Koyanagi, Y., Blauvelt, A. et al. 2013. Antimicrobial peptide LL-37

produced by HSV-2-infected keratinocytes enhances HIV infection of Langerhans cells.

Cell. Host. Microbe. 13, 77-86.

Burkard, C., Bloyet, L.M., Wicht, O., van, Kuppeveld F.J., Rottier, P.J., de, Haan C.A., and

Bosch, B.J. 2014. Dissecting virus entry: replication-independent analysis of virus binding,

internalization, and penetration using minimal complementation of ホイ-galactosidase. PLoS.

One. 9, e101762.

Oda, T., Yanagisawa, H., and Kikkawa, M. 2015. Detailed structural and biochemical

characterization of the nexin-dynein regulatory complex. Mol. Biol. Cell. 26, 294-304.

Ribeiro, C.M., Sarrami-Forooshani, R., Setiawan, L.C., Zijlstra-Willems, E.M., van,

Hamme J.L., Tigchelaar, W., van, der Wel N.N., Kootstra, N.A., Gringhuis, S.I., and

Geijtenbeek, T.B. 2016. Receptor usage dictates HIV-1 restriction by human TRIM5alpha

in dendritic cell subsets. Nature. 540, 448-452.

Stewart-Jones, G.B., Soto, C., Lemmin, T., Chuang, G.Y., Druz, A., Kong, R., Thomas,

P.V., Wagh, K., Zhou, T., Behrens, A.J. et al. 2016. Trimeric HIV-1-Env Structures Define

Glycan Shields from Clades A, B, and G. Cell. 165, 813-826.

Doebel, T., Voisin, B., and Nagao, K. 2017. Langerhans Cells - The Macrophage in 
Dendritic Cell Clothing. Trends. Immunol. 38, 817-828.

Zheng, S.Q., Palovcak, E., Armache, J.P., Verba, K.A., Cheng, Y., and Agard, D.A. 2017.

MotionCor2: anisotropic correction of beam-induced motion for improved cryo-electron

microscopy. Nat. Methods. 14, 331-332.

Bermejo-Jambrina, M., Eder, J., Helgers, L.C., Hertoghs, N., Nijmeijer, B.M.,

Stunnenberg, M., and Geijtenbeek, T.B.H. 2018. C-Type Lectin Receptors in Antiviral

Immunity and Viral Escape. Front. Immunol. 9, 590.

Goddard, T.D., Huang, C.C., Meng, E.C., Pettersen, E.F., Couch, G.S., Morris, J.H., and

Ferrin, T.E. 2018. UCSF ChimeraX: Meeting modern challenges in visualization and

analysis. Protein. Sci. 27, 14-25.

Nakane, T., Kimanius, D., Lindahl, E., and Scheres, S.H. 2018. Characterisation of

molecular motions in cryo-EM single-particle data by multi-body refinement in RELION.

Elife. 7, e36861.

Gonelli, C.A., Khoury, G., Center, R.J., and Purcell, D.F.J. 2019. HIV-1-based Virus-like

Particles that Morphologically Resemble Mature, Infectious HIV-1 Virions. Viruses. 11, 507.

Heredia, J.D., Park, J., Choi, H., Gill, K.S., and Procko, E. 2019. Conformational 
Engineering of HIV-1 Env Based on Mutational Tolerance in the CD4 and PG16 Bound

States. J. Virol. 93, e00219-00219.

Postma, M., and Goedhart, J. 2019. PlotsOfData-A web app for visualizing data together

with their summaries. PLoS. Biol. 17, e3000202.

Pan, J., Peng, H., Chen, B., and Harrison, S.C. 2020. Cryo-EM Structure of Full-length

HIV-1 Env Bound With the Fab of Antibody PG16. J. Mol. Biol. 432, 1158-1168.

Thornton, S.M., Samararatne, V.D., Skeate, J.G., Buser, C., Luhen, K.P., Taylor, J.R., Da,

Silva D.M., and Kast, W.M. 2020. The Essential Role of anxA2 in Langerhans Cell

Birbeck Granules Formation. Cells. 9, 974.

Kimanius, D., Dong, L., Sharov, G., Nakane, T., and Scheres, S.H.W. 2021. New tools for automated cryo-EM single-particle analysis in RELION-4.0. Biochem. J. 478, 4169-4185.

Lempp, F.A., Soriaga, L.B., Montiel-Ruiz, M., Benigni, F., Noack, J., Park, Y.J., Bianchi, S., Walls, A.C., Bowen, J.E., Zhou, J. et al. 2021. Lectins enhance SARS-CoV-2 infection and influence neutralizing antibodies. Nature. 598, 342-347.

Rhodes, J.W., Botting, R.A., Bertram, K.M., Vine, E.E., Rana, H., Baharlou, H., Vegh, P., O'Neil, T.R., Ashhurst, A.S., Fletcher, J. et al. 2021. Human anogenital monocyte-derived dendritic cells and langerin+cDC2 are major HIV target cells. Nat. Commun. 12, 2147. 
bioRxiv preprint doi: https://doi.org/10.1101/2022.02.24.481763; this version posted February 25, 2022. The copyright holder for this preprint (which was not certified by peer review) is the author/funder, who has granted bioRxiv a license to display the preprint in perpetuity. It is made available under aCC-BY 4.0 International license.

A

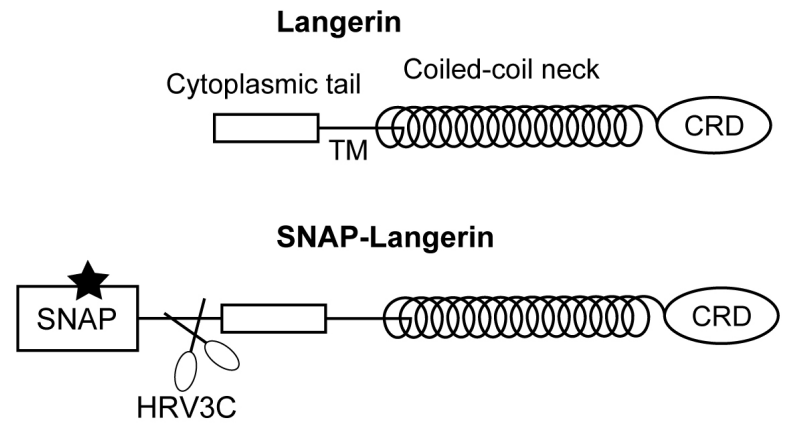

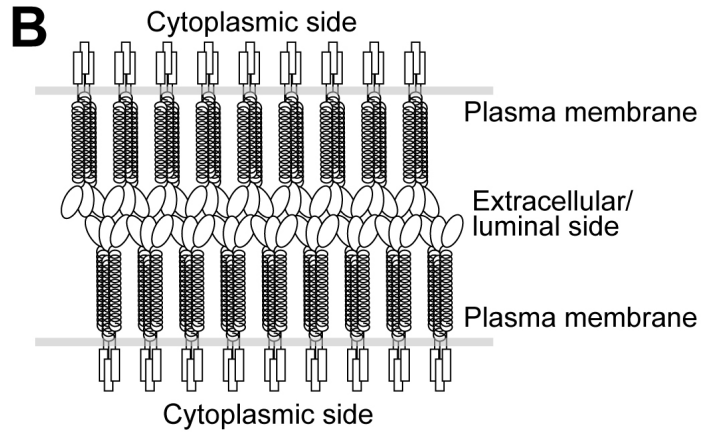
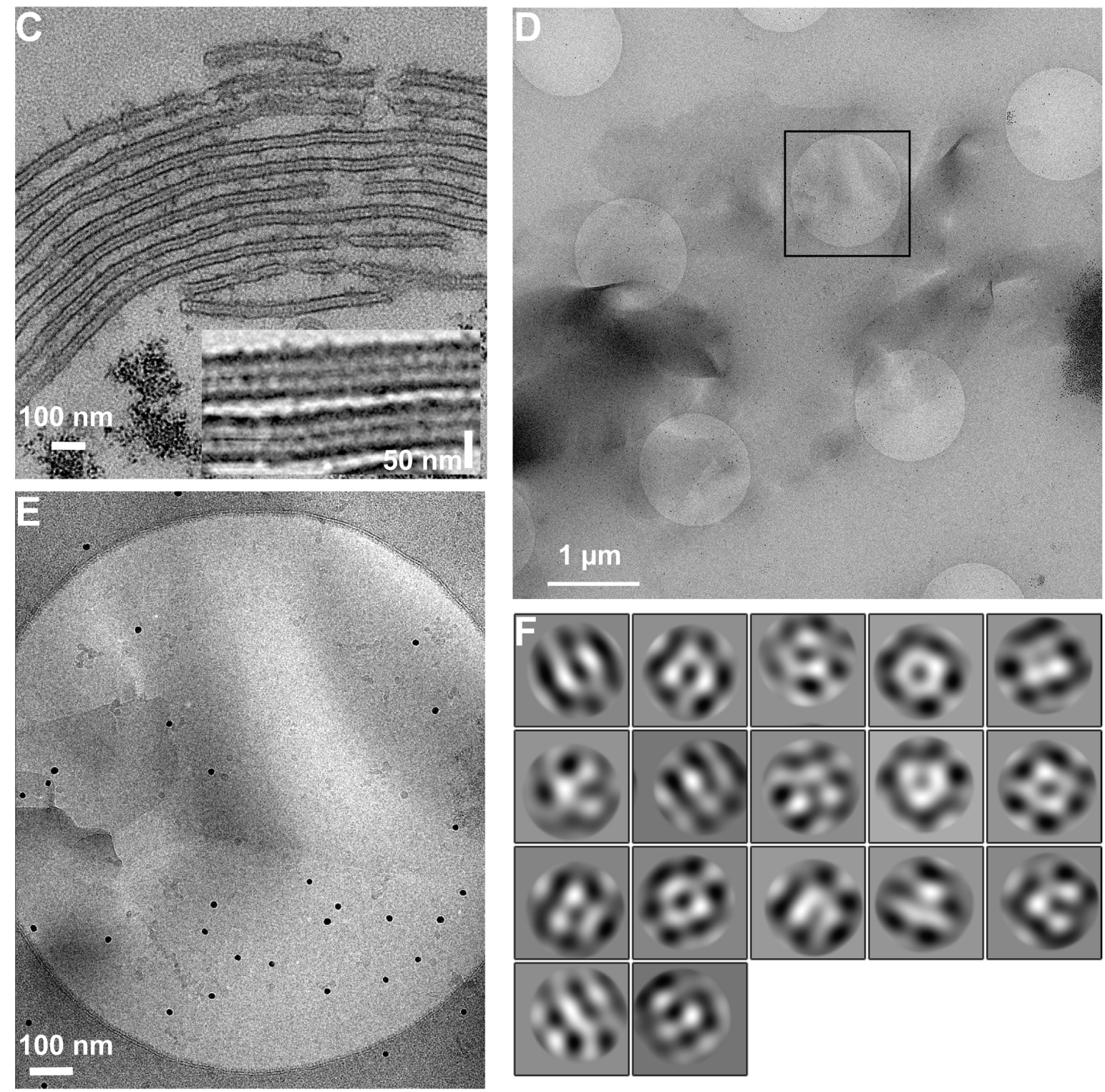

Figure 1 
Figure 1: Isolation of Birbeck granules. (A) Domain organization of langerin. Human langerin is a 328-amino acids protein composed of an N-terminal cytoplasmic tail, a transmembrane domain (TM), a coiled-coil neck, and a C-terminal carbohydrate-recognition domain (CRD). We fused a SNAP-tag at the N-terminus of langerin and inserted an HRV3C protease cleavage site between the tag and the langerin sequences. For streptavidin-mediated precipitation of Birbeck granules, the SNAP-tag was biotinylated (star). (B) Model of langerin oligomerization within Birbeck granules. Langerin trimers bind to each other faceto-face via the CRDs, bringing the two layers of the plasma membrane closer together. (C) Birbeck granules formed in $293 \mathrm{~T}$ cells overexpressing langerin. Addition of yeast mannan induced the formation of Birbeck granules a few micrometers long. Inset shows a magnified view of Birbeck granules. (D) Cryo-electron microscopy of isolated Birbeck granules. Black square indicates the position of the close-up view shown in E. (E) Wavy lamellar structure of the Birbeck granule. Black dots were gold nanoparticles used as fiducial markers. (F) Class averages of the projection images of Birbeck granules. The image dimension is $34 \mathrm{~nm}^{2}$. Although 2D classification did not converge well due to the continuity of the structure, some classes showed a porous structure with a honeycomb-like lattice. 
bioRxiv preprint doi: https://doi.org/10.1101/2022.02.24.48176. this version posted February 25, 2022. The copyright holder for this preprint (which was not certified by peer review) is the author/funder, who has granted bioRxiv a license to display the preprint in perpetuity. It is made available under aCC-BY 4.0 International license.

A
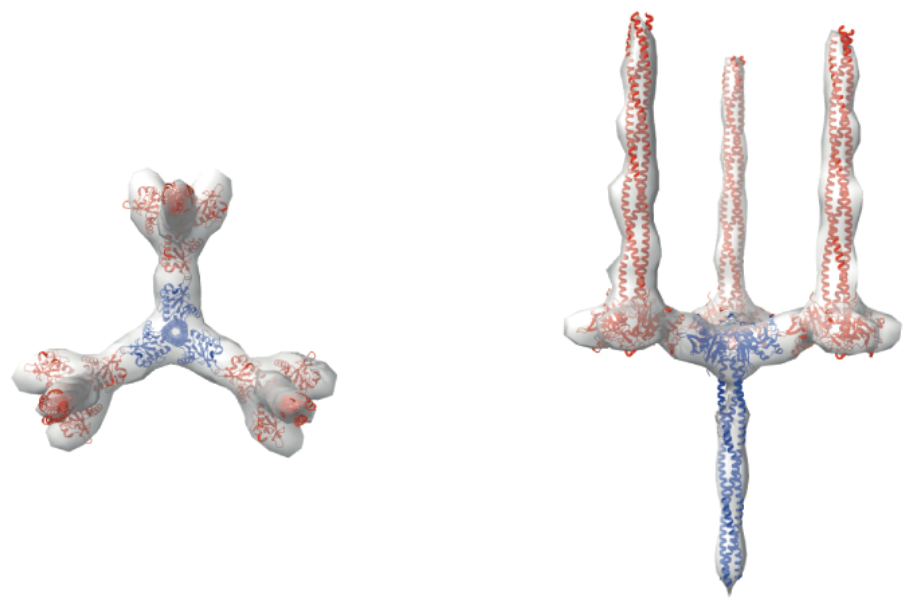

B

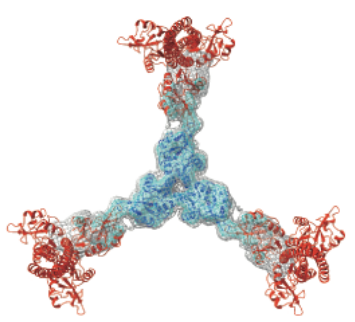

C
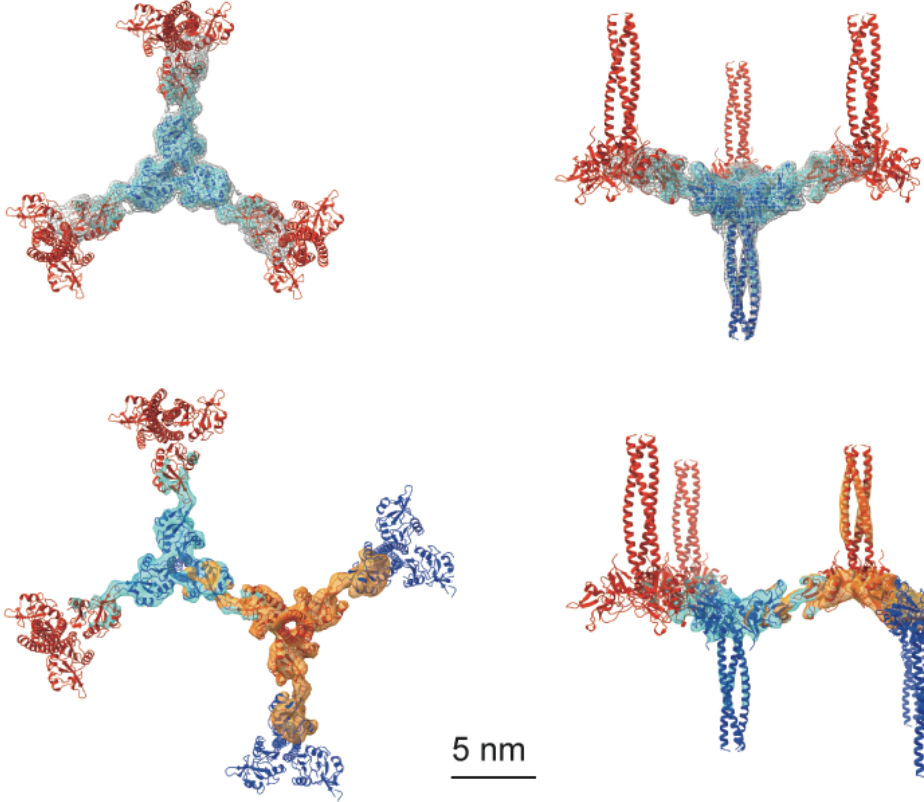

D

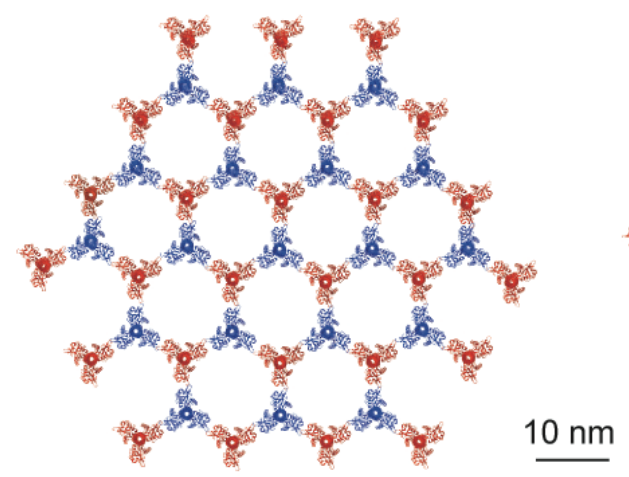

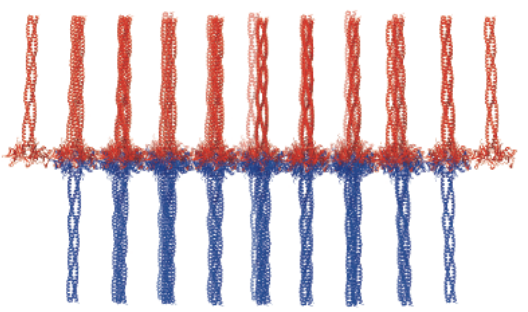

Figure 2 
Figure 2: Averaged subtomograms of Birbeck granules. (A) Result of the initial 3D

refinement. The central langerin trimer (blue) binds to the three inverted langerin trimers

(red). (B) Result of the focused refinement. Improved resolution (6.4 $\AA$ ) allowed secondary structure modeling. The gray mesh and the cyan surface indicate low and high threshold isosurfaces, respectively. (C) Result of the two-body refinement. The second body (orange) was refined with respect to the first body (cyan). (D) Honeycomb model of the langerin lattice within Birbeck granules. This honeycomb lattice is an ideal model, with the assumption of structural uniformity. (A-D) left, top views; right, side views. 
bioRxiv preprint doi: https://doi. org/10.1101/2022 02.24.481763: this version posted February 25, 2022 The copyright holder for this preprint (which was not certified by peer review) is the author/funder, who has granted bioRxiv a license to display the preprint in perpetuity. It is made available under aCC-BY 4.0 International license.

A
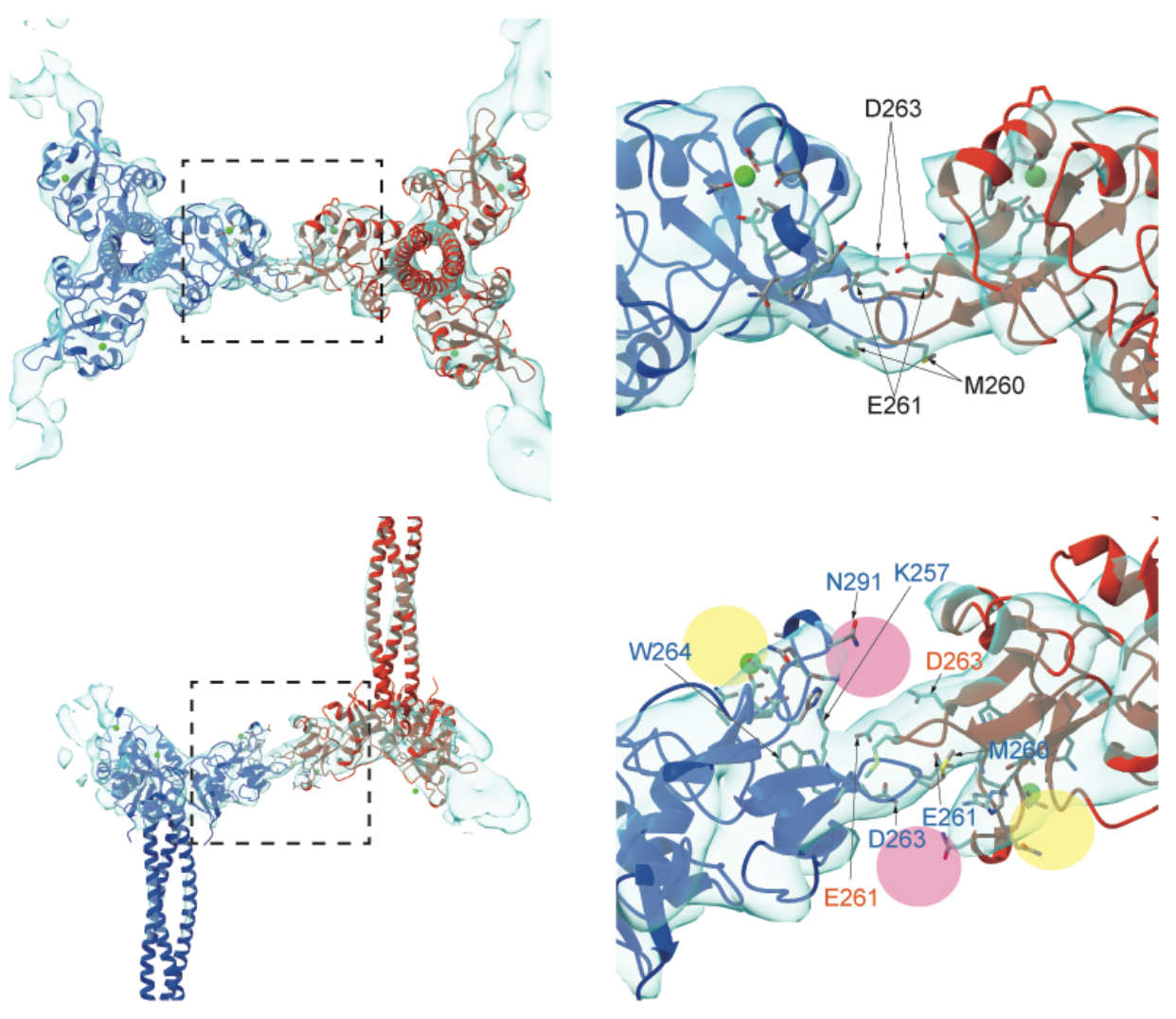

B
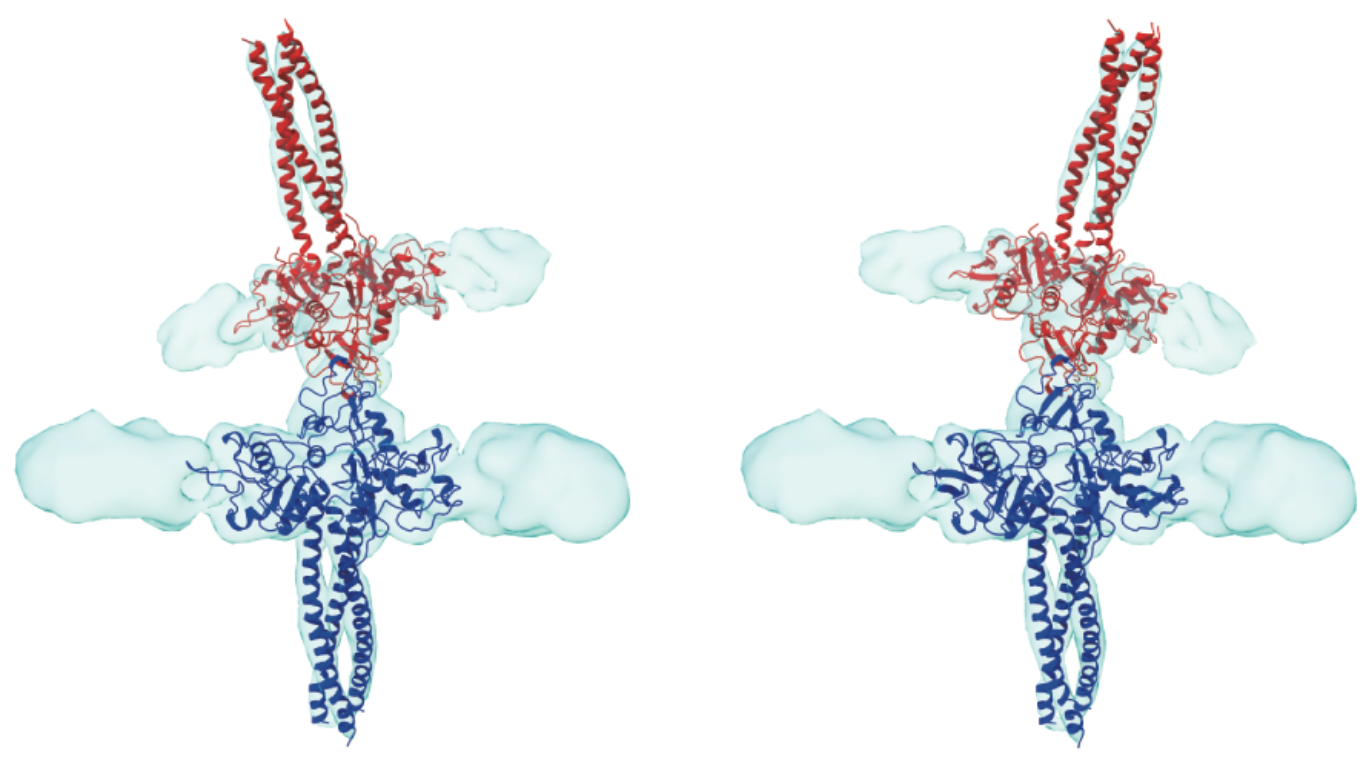

Figure 3 
Figure 3: Binding interface between two langerin trimers. (A) Two langerin trimers interact via the loops at residues 258-262. Amino acid codes and residue numbers are indicated. Yellow and pink circles indicate the primary and secondary carbohydrate-binding sites. The orientations of the side chains are speculative because the resolution of these maps (6.4 $\AA$ ) was insufficient for precise modeling. Calcium ions (green) were placed based on the crystal structure (PDB ID: 3KQG). Top and side views are displayed in the top and bottom rows, respectively. Broken squares indicate the positions of the magnified views on the right. (B) Flexibility between the two trimers. The first eigenvector of the structural variation of the inverted trimer (red) relative to the upright trimer (blue) shows an approximately $30^{\circ}$ rotation about the loop 258-262 (see Movie S2). 
A

${ }_{M}^{260} \operatorname{MEGD}^{263}(\mathrm{WT})$

MRGD

MRGK

ARGK
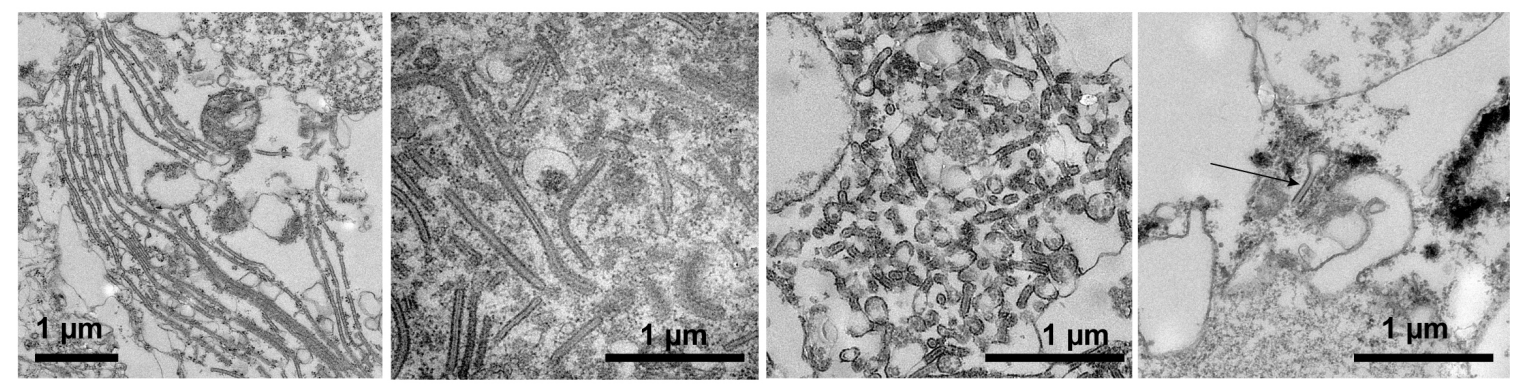

B

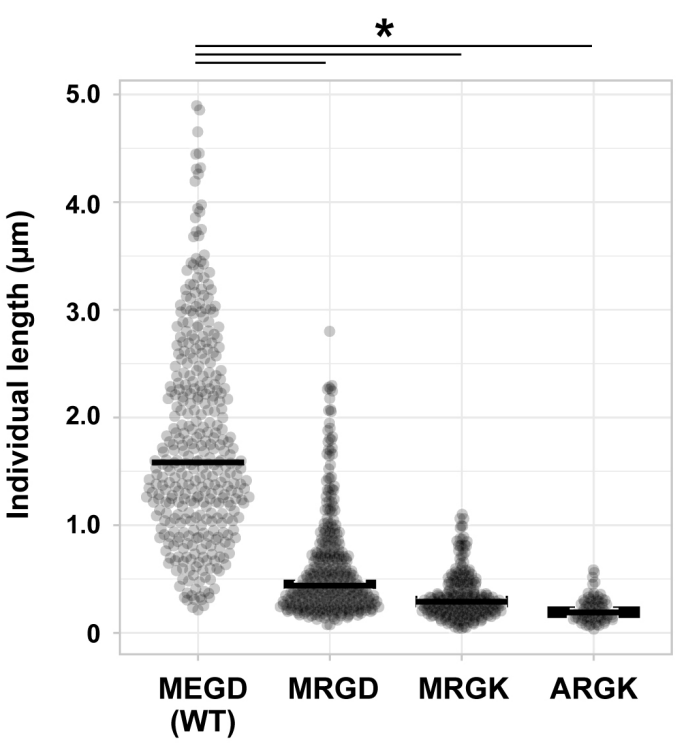

C

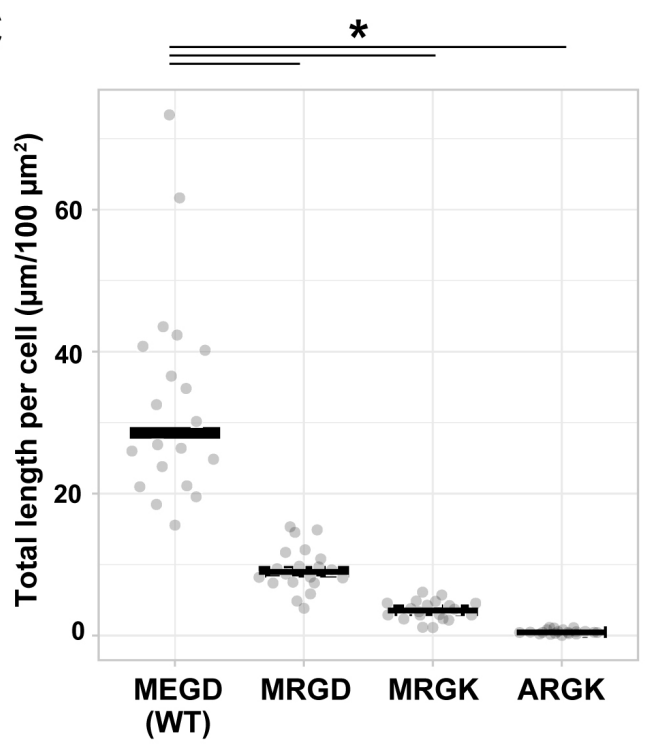

Figure 4

Figure 4: Mutations to the loop 258-262 disrupted Birbeck granule formation. (A) Ultra-

thin electron microscopy of Birbeck granules induced by yeast mannan in langerinoverexpressing 293T cells. Residues at 260-263 (Met-Glu-Gly-Asp, MEGD) were mutated to Met-Arg-Gly-Asp (MRGD), Met-Arg-Gly-Lys (MRGK), or Ala-Arg-Gly-Lys (ARGK).

The WT image has a lower magnification than the others in order to show the entire Birbeck 
granules. (B, C) Quantification of Birbeck granule formation. Horizontal lines indicate the median values. Asterisks indicate statistically significant differences $(p<0.01)$ calculated using Bonferroni-corrected Student's $t$-tests. (B) Lengths of individual Birbeck granules were measured. $\mathrm{N}=386$ (WT), 348 (MRGD), 233 (MRGK), and 70 (ARGK). (C) Sum of the length of Birbeck granules per $100 \mu \mathrm{m}^{2}$ cell area. Cross-sections of cells with nuclei of $>5$ $\mu \mathrm{m}$ in diameter were selected and the total lengths of the Birbeck granules were measured within the cells. $\mathrm{N}=20$ for all four samples. 
A

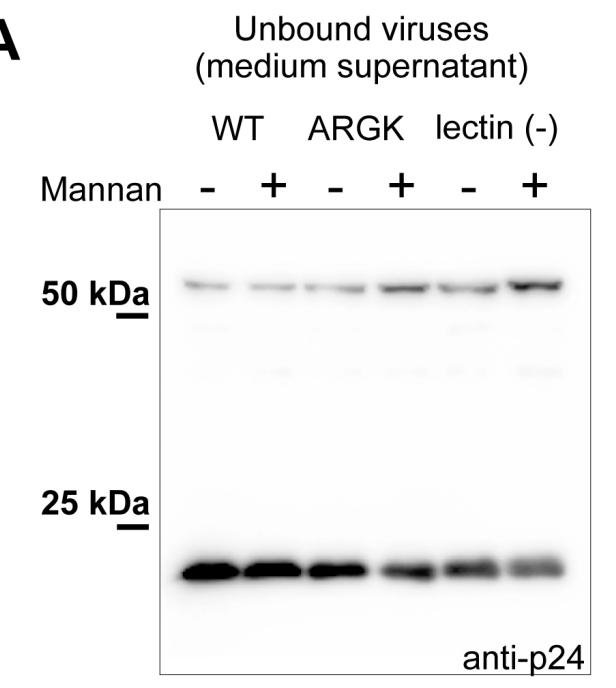

Unbound viruses
(medium supernatant)

Attached viruses

(EDTA wash)

WT ARGK lectin (-)

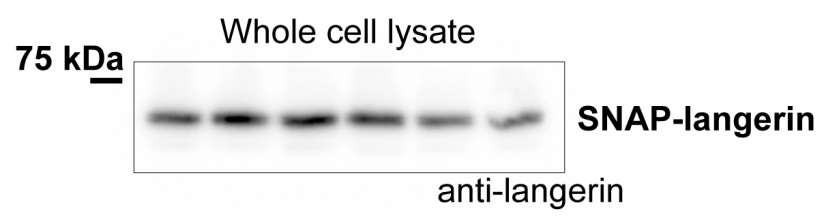

B
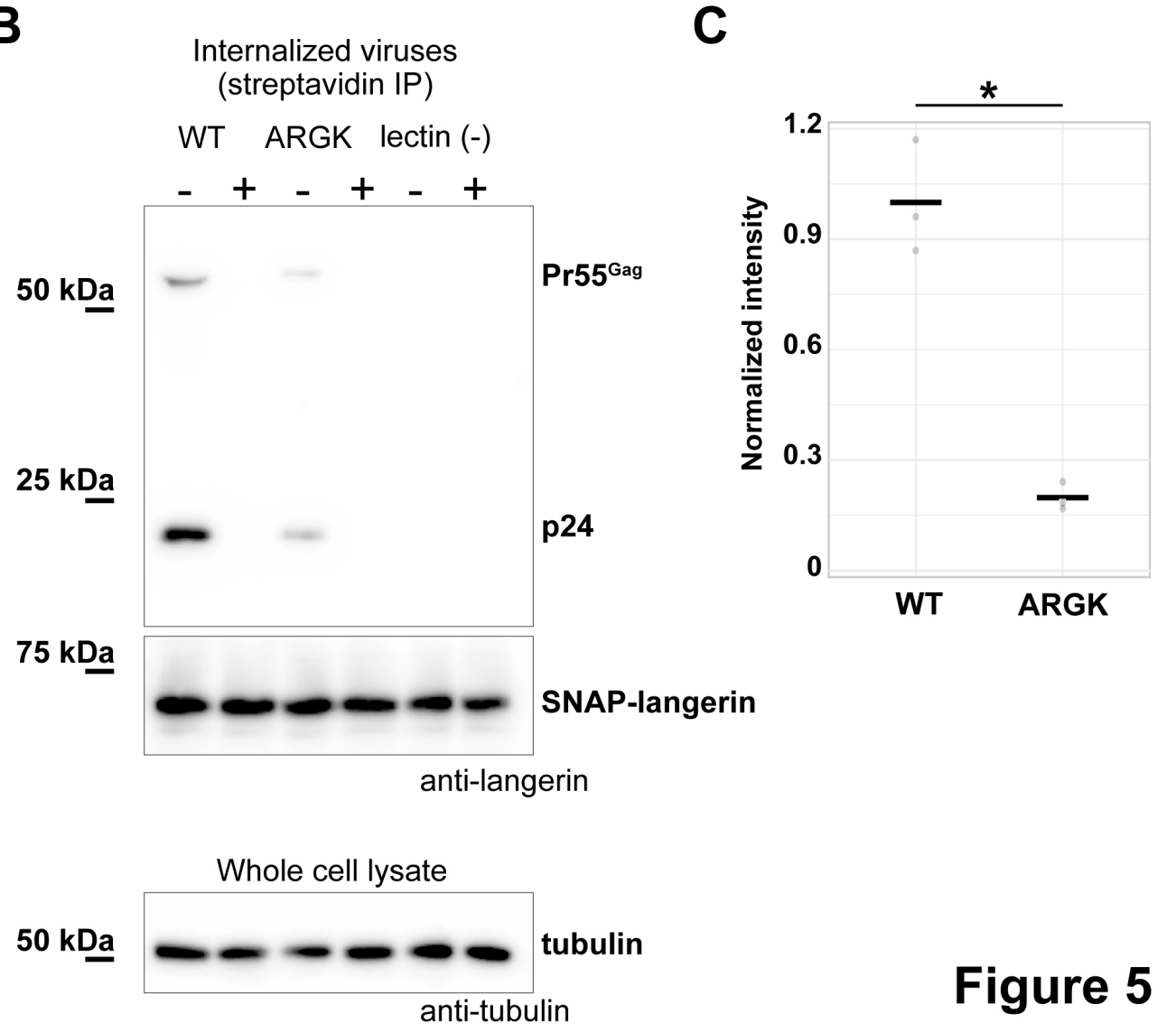

Figure 5 
Figure 5: Mutation to the loop 258-262 inhibited the internalization of pseudoviruses.

HIV-1 pseudoviruses were added to langerin-expressing 293T cells. Yeast mannan (10 $\mu \mathrm{g} / \mathrm{ml}$ ) was added to block the lectin-dependent binding of pseudoviruses. A langerin mutant lacking calcium binding ability (lectin (-)) was used as the negative control. (A) Immunoblots of pseudoviruses attached to the cell surface. Unbound and attached viruses were collected from the supernatant of the culture medium and TBS-EDTA buffer, respectively. Samples of unbound viruses were diluted 50-fold to adjust the band intensities. The expression levels of SNAP-tagged langerin show that the numbers of transfected cells were approximately the same in each experiment. Pr55 gag and p24 indicate unprocessed and fully-processed capsid proteins, respectively. (B) Immunoblots of internalized pseudoviruses. Birbeck granules were isolated by precipitation using streptavidin-agarose, and intracellular viruses and langerin were detected by their respective antibodies. Tubulins in the whole-cell lysates were detected for loading controls. (C) Quantification of immunoblots in B. Amounts of internalized virus in the absence of mannan were compared by normalizing the intensities of p24 bands to those of corresponding langerin bands. Horizontal lines indicate the mean. Asterisks indicate statistically significant differences $(p=0.0076)$ calculated using a paired Student's $t$-test $(\mathrm{N}=3)$. 
A
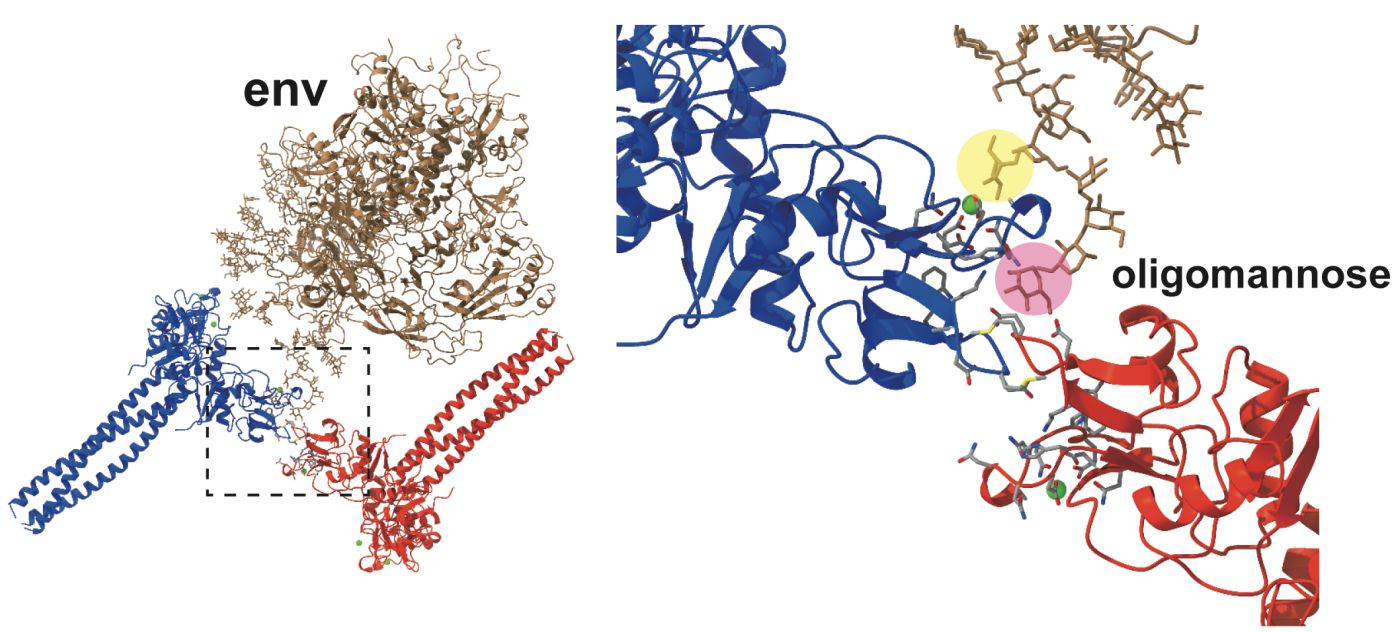

B

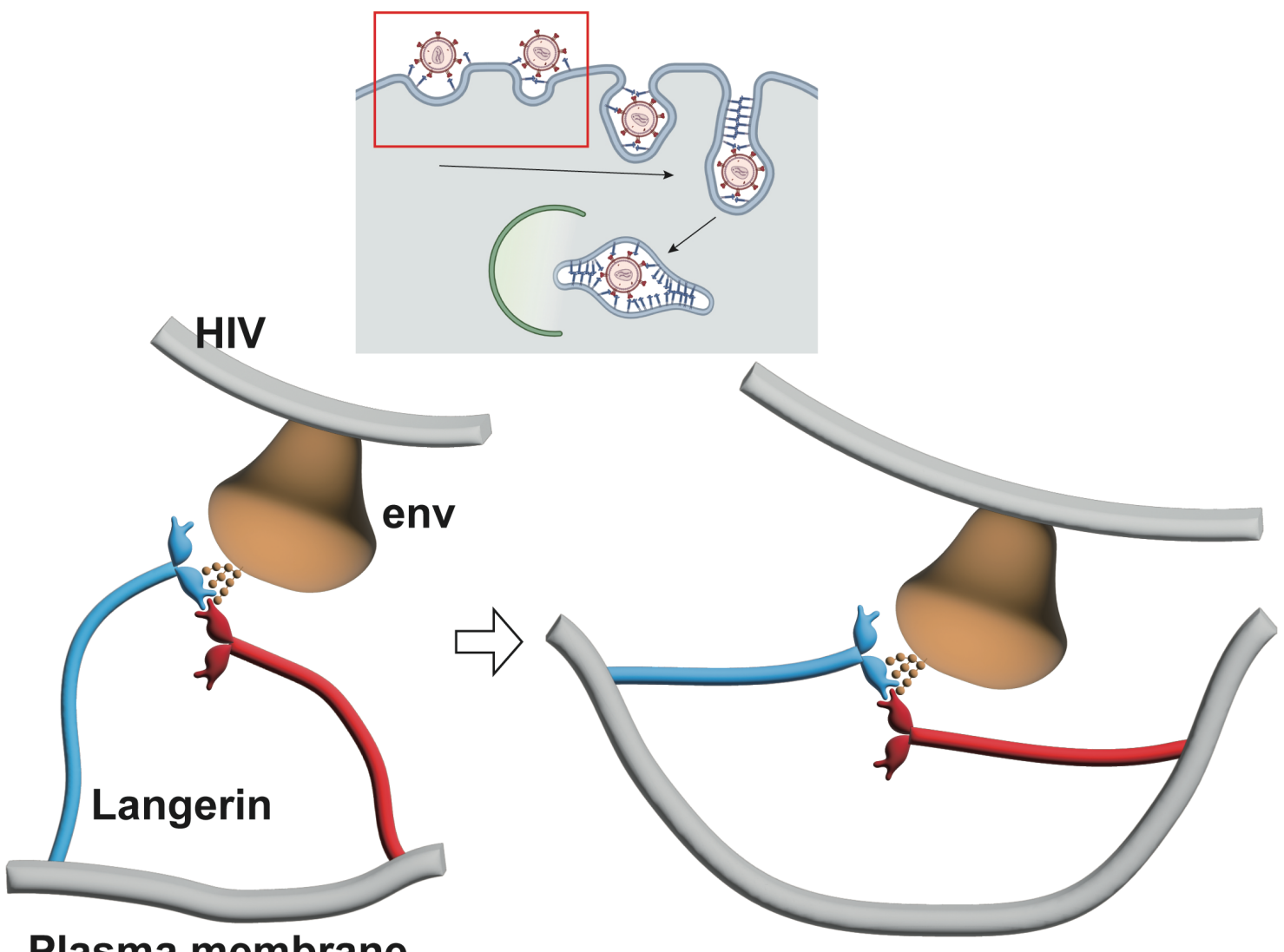

Plasma membrane

Figure 6

Figure 6: Model of HIV internalization by langerin. (A) Glycosylated structure of HIV-1 
envelope (env, gray) (Constructed based on PDB ID: 5FYJ and 6ULC) bound to the langerin trimer complex. Branches of the high-mannose oligosaccharide interacting with the primary (yellow) and secondary (pink) carbohydrate-binding sites. The broken square indicates the position of the close-up view on the right. (B) Schematics of HIV internalization by langerin. The inset on the top shows an overview of antigen processing within a Langerhans cell. Viruses are captured by langerin, internalized into a Birbeck granule, and degraded by the autophagic system. The red square indicates the viral entry process described in the lower panels. The high-mannose oligosaccharide on HIV-1 env first binds to the primary carbohydrate-binding site, and one of the branches of the oligo-mannose weakly interacts with the secondary binding site. The flexible neck region allows docking of the loop 258-262 of an adjacent trimer into the secondary carbohydrate-binding cleft, reinforcing the binding of the oligo-mannose. As the elastic neck regions straighten, the plasma membrane is deformed into a lamellar invagination, which internalizes the virus. 
bioRxiv preprint doi: https://doi. org/10.1101/2022.02.24.481763 this version posted February 25, 2022 The copyright holder for this preprint (which was not certified by peer review) is the author/funder, who has granted bioRxiv a license to display the preprint in perpetuity. It is made available under aCC-BY 4.0 International license.

A

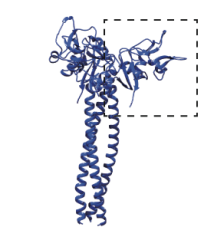

Langerin

DC-SIGN
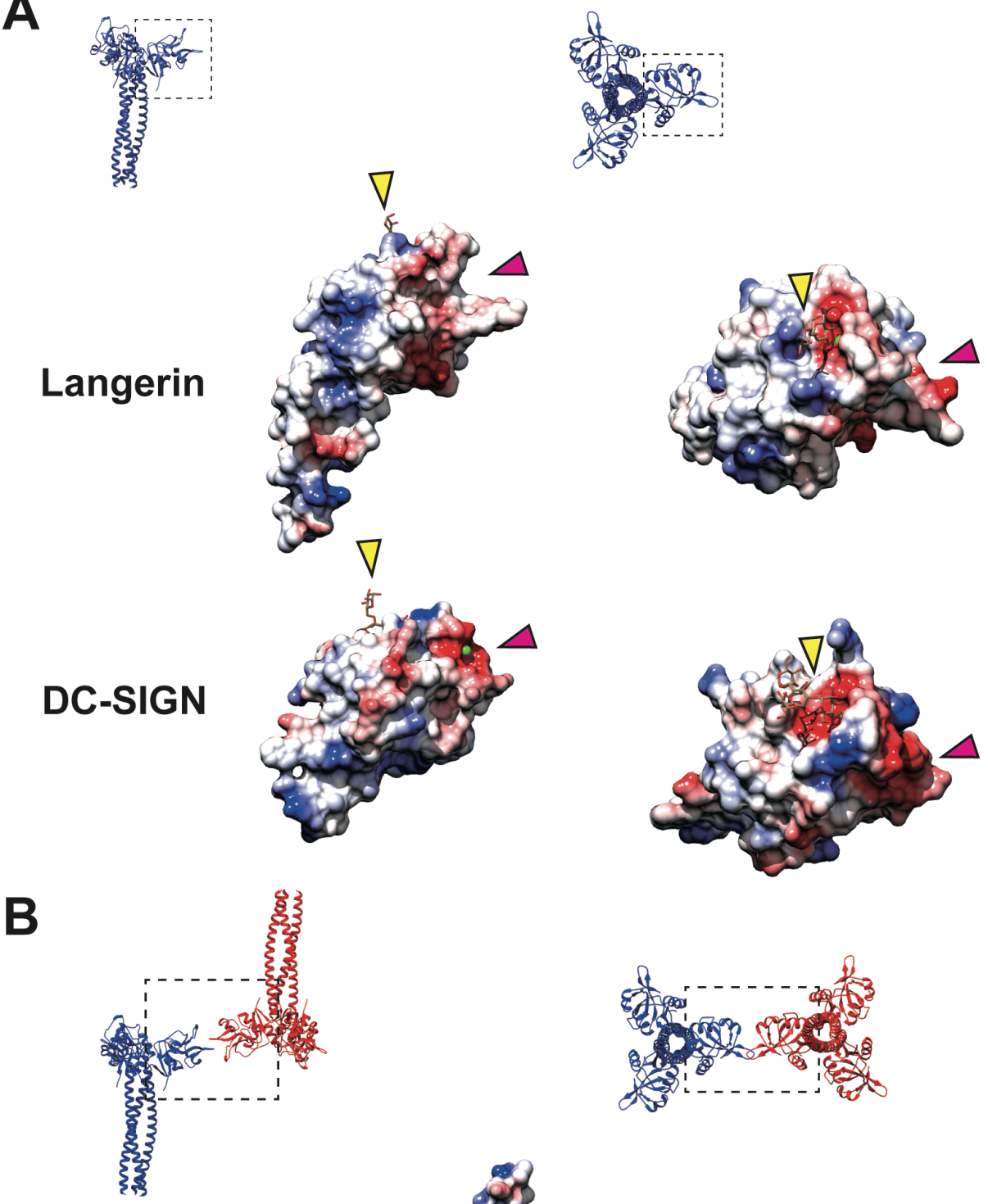

Figure 7 
Figure 7: Coulombic surfaces of the carbohydrate-binding sites. (A) Comparison of the surface charge between the CRDs of langerin and DC-SIGN (PDB ID: 1SL4). Blue and red coloring indicates positive and negative charges, respectively. The primary and secondary carbohydrate-binding sites are indicated by yellow and pink arrowheads, respectively. The region of DC-SIGN corresponding to the secondary carbohydrate-binding sites of langerin is occupied by calcium ions (green) and does not form a cleft. (B) Surface model of the langerin trimer complex. Mannose ligands are placed based on a previously proposed model (Chatwell et al., 2008). (A-B) Broken squares indicate the position and the orientation of the side (left) and the top (right) views. Oligosaccharide ligands are shown in gray. 